\title{
CBCT Visualization of Furcation Perforation Repair Materials Using Different Voxel Sizes
}

\author{
Ayse Isil Orhan ${ }^{1}$, Pelin Tufenkci ${ }^{\circledR}$, Aysenur Oncu ${ }^{3}$, Sevinc Sevgi ${ }^{3}$, Berkan Celikten ${ }^{3} \oplus$, Kaan Orhan $^{4}$ \\ ${ }^{1}$ Ankara Yıldırım Beyazıt University, Faculty of Dentistry, Department of Pediatric Dentistry, Ankara, Turkey. \\ ${ }^{2}$ Hatay Mustafa Kemal University, Faculty of Dentistry, Department of Endodontics, Hatay, Turkey. \\ ${ }^{3}$ Ankara University, Faculty of Dentistry, Department of Endodontics, Ankara, Turkey. \\ ${ }^{4}$ Ankara University, Faculty of Dentistry, Department of Oral and Maxillofacial Radiology, Ankara, Turkey. \\ Correspondence Author: Ayse Isil Orhan \\ E-mail: isilcihan@yahoo.com
}

Received: $19.06 .2021 \quad$ Accepted: 28.06 .2021

\begin{abstract}
Objective: Three-dimensional cone-beam computed tomography is gaining popularity as an imaging modality aiding the performance of difficult endodontic treatment procedures. For this reason, we assessed the visualization of bioactive furcal perforation repair materials in an ex vivo study using CBCT with different voxel sizes and determined which voxel size yielding the best images with least artefacts. Visualization of endodontic restorative material is affected by the appearance of various artifacts. This study was conducted to evaluate the CBCT visualization of three perforation repair materials using five different voxel sizes.

Methods: This study was performed with 84 mandibular molars that had been extracted for various reasons. All samples were measured with a digital caliper (Digimess, São Paulo, Brazil), marked at $3 \mathrm{~mm}$ above the cementoenamel junction, and decoronized at this line. The root segments were amputated at $3 \mathrm{~mm}$ below the furcation site using diamond discs under water cooling. The openings of the pulp chamber created, following the furcation defects formed. Biodentine, MTA, and EndoSequence were mixed following the manufacturers' instructions and applied to the perforation sites. Five image sets were obtained: 75 micron, 100 micron, 150 micron, 200 micron, and 400 micron. 2 examiners were evaluated the images independently.

Results: The best image quality for all repair materials obtained in 100 micron. Image clarity of repair materials showed no significant difference among voxel sizes of 75 and 150 micron. Image quality was significantly reduced at 200 and 400 micron voxel sizes, and the worst quality was obtained at 400 micron voxels in all groups.

Conclusions: $\mathrm{CBCT}$ imaging can be used to examine endodontic repair materials with adjustment of the effective radiation dose rate and selection of the appropriate voxel size.

Keywords: Bioactive aterials, Perforation, Voxel size
\end{abstract}

\section{INTRODUCTION}

Perforations and defects in the furcation region affect prognoses following endodontic treatment. Furcation perforations are iatrogenic gaps between the pulp chamber and periodontal ligament; they can cause complications during endodontic treatment and subsequent periodontitisrelated lesions (1). Defects on the floor of the pulp chamber lead to periodontal ligament and bone destruction, followed by an inflammatory process that can lead to tooth loss (2). The success of endodontic treatment of a tooth with a furcation perforation depends on furcal defect size, location, the time spent closing it, and the properties of the materials used (3). Many repair materials have been developed and used for perforation closure, including calcium silicate-based cements, such as mineral trioxide aggregate (MTA; Dentsply
Maillefer, Ballaigues, Switzerland) (4). MTA is commonly used as a repair material, but it has several limitations (5).

Biodentine (Septodont, Niederkassel, Germany), EndoSequence Materials (Brasseler USA, Savannah, GA, USA) are newly developed bioactive materials. Bioceramics are biocompatible ceramic compounds obtained in situ and in vivo through various chemical processes (6). They are resistant to irrigation and antibacterial, with osteogenetic effects and short application times (7). Biodentine was stated to be improved behaviour properties and takes less time to prepare than MTA. Its physical properties are very similar to dentine and the risk of tooth discoloration is low (8). Recently, EndoSequence was designed for use as root repair material. This premixed material is provided as a condensed mass or 
pre-filled syringe and is easy to manipulate; it has excellent biological and mechanical properties and a high degree of biocompatibility. In addition, it is hydrophilic, radiopaque, osteogenic, and insoluble. It is used for pulp capping and root repair procedures (9). Our understanding of the radiological properties of bioceramic materials is limited by numerous factors (10). Two-dimensional x-rays are inadequate for the analysis and handling of furcation defects. Cone-beam computed tomography (CBCT) is used for endodontic identification, management, prognosis, as it enables the three-dimensional (3D) identification of bony margins and defect sites (11). Voxel size has important effects on CBCT evaluation quality. A voxel is the smallest element of a $3 D$ image. In CBCT imaging, voxels are usually isotropic and range from $75-400$ micron. The isotropic geometry of the voxels allows for identical image resolution on different planes (2). Visualization of endodontic restorative material is affected by the appearance of various artifacts. Although these image defects cannot be eliminated using current technology, the quality can be improved by changing constraints such as the FOV and voxel sizes (12). Thus, this study was conducted to evaluate the CBCT visualization of three perforation repair materials using five different voxel sizes.

\section{METHODS}

\subsection{Sample Selection}

84 mandibular molars were used for this study that had been extracted for various reasons. Ankara University Local Ethical Committee (IRB approval number: 07/10) approved the study. Included teeth were intact or with minimal caries, also had closed apexes and separate roots. Radiographs of all teeth were taken from different directions and examined; teeth with calcified pulp chambers or pulp stones were excluded. Residual debris and soft tissue on the teeth were removed using a periodontal curette, and the teeth were kept in distilled water containing $0.1 \%$ thymol crystal at room temperature until use. All samples were measured with a digital caliper (Digimess, São Paulo, Brazil), marked at $3 \mathrm{~mm}$ above the cementoenamel junction, and decoronized at this line. The root segments were amputated at $3 \mathrm{~mm}$ below the furcation site using diamond discs under water cooling. The openings of the pulp chamber created. Necrotic pulp tissue removed and the cavities were rinsed with sodium hypochlorite. Dentin thickness in the furcation area was measured using a calliper, and teeth with dentine thicknesses of 2.0-2.5 mm were included in the study. The perforations were formed in the furcation areas using a long round drill with water cooling. The perforation areas were irrigated using saline to remove residual dentin. To simulate clinical conditions, the samples were placed in sponges soaked in saline in plastic cylinders. The teeth were then divided randomly into three groups. Biodentine, MTA-Angelus (Angelus Indústria de Produtos Odotontológicos S/A, Londrína, PR, Brazil), and EndoSequence Root Repair Material were mixed according to manufacturer directions and placed over the perforation sites for respective groups using a plugger and dental loupe. The materials were condensed by applying a small amount of pressure. Excess filling material was removed with a thin probe, and a damp cotton pellet was then placed into pulp to harden the material. The samples left at $37^{\circ} \mathrm{C}$ with $100 \%$ moisture for 7 days to allow the materials to harden.

\subsection{Scanning Protocol}

Each tooth positioned on molar tooth sockets in a dry human mandible. The mandible was properly covered with wax to simulate soft tissue. The mandible then scanned using Planmeca ProMax 3D CBCT (Planmeca Oy, Helsinki, Finland). The scanner offers multiple fields of view, allowing the dentist to select the optimum scan on a case-by-case basis. Images were obtained using a $5 \times 5.5 \mathrm{~cm}$ FOV (75 micron and 100 micron), a $5 \times 5.5 \mathrm{~cm}$ FOV (150 micron), a $10 \times 5.5 \mathrm{~cm}$ FOV (200 micron and 400 micron) with isotropic voxels. All scans were taken at pre-settings of the machine; $90 \mathrm{kVp}, 10 \mathrm{~mA}, 17$ $\mathrm{s}$ and $90 \mathrm{kV}, 10 \mathrm{~mA}, 15 \mathrm{~s}$ for $(75,100$, and 150 micron), 96kVp, $12 \mathrm{~mA}, 18 \mathrm{~s}$ for (200 micron and 400 micron) voxels.

\subsection{CBCT Evaluations}

Each scan was evaluated by 2 observers with a mean of 10 years of experience using the unit's software (Planmeca Romexis 4.2). Before evaluations, the observers were pretrained on for the usage of the software in a special session. The observers were calibrated for the study. The images were anonymized during evaluation of the materials. Observers scored clarity of the image of the materials. They were also asked to grade overall image quality on a 1-to-5 point scale based on the visibility of perforation repair materials of different voxel sizes (1: very low, 2: low, 3: moderate, 4: high, 5: very high quality).

The images evalautions were made within one week again, and all images again re-evalueted after 2 months.

\subsection{Statistical Analysis}

The data analyzed with SPSS (ver. 22; Chicago, IL, USA). Receiver operating characteristic (ROC) curves were used for each observers' evaluations. A $p$ value less than 0.05 was considered as significant. Inter-examiner reliability evalauted with Kappa Cohen's statistical analysis. Weighted kappa coefficients were calculated to assess both intra and interobserver agreements for each image set. Weighted kappa values were interpreted according to the guidelines of Landis and Koch, as adapted by Altman ( $\leq \leq 0.20$, very low; $\mathrm{k}=$ $0.20-0.40$, low; $\mathrm{k}=0.41-0.60$, moderate; $\mathrm{k}=0.61-0.80$, high; and $k=0.81-1.00$, very high quality). The normality of the variables was analysed using the Shapiro-Wilk test. Moreover, three-way ANOVA was used with Bonferroni correction. 


\section{RESULTS}

The inter-examiner reliability showed a high agreement of 90.4\% (95\% Cl 0.773-0.846) between the two observers (KO, BC) which showed no significant difference between observers.

Three-way analysis of variance revealed significant effects of the voxel and material factors. The voxel and material factors had significant effects $(p<0.05)$. No interaction between factors was observed.

The best image quality for all repair materials obtained at 100 micron. There was no significant difference among 75 micron and 150 micron for visualization of repair materials.

Image quality of repair materials was significantly reduced at 200 and 400 micron and the worst quality was obtained at 400 micron voxels in all groups (Table 1).

Table 1. Showing the mean values and standard deviation of the endodontic repair materials according to voxel sizes.

\begin{tabular}{|c|c|c|c|}
\hline Restorati & \multirow{2}{*}{ Endosequence } & \multirow{2}{*}{ Biodentine } & \multirow{2}{*}{ MTA-Angelus } \\
\hline Voxel sizes & & & \\
\hline 75 micron & $4,167 \pm 0,637^{a A}$ & $4,042 \pm 0,624^{\mathrm{aAB}}$ & $3,917 \pm 0,503^{\mathrm{aA}}$ \\
\hline 100 micron & $4,417 \pm 0,653^{\mathrm{aB}}$ & $4,375 \pm 0,575^{a \mathrm{~B}}$ & $4,000 \pm 0,0^{\mathrm{aA}}$ \\
\hline 150 micron & $4,000 \pm 0,417^{\mathrm{aBC}}$ & $3,958 \pm 0,690^{\mathrm{AB}}$ & $4,000 \pm 0,0^{\mathrm{aA}}$ \\
\hline 200 micron & $3,250 \pm 0,442^{\mathrm{ac}}$ & $3,542 \pm 0,883^{\mathrm{aA}}$ & $3,583 \pm 0,503^{2 \mathrm{~A}}$ \\
\hline 400 micron & $1,833 \pm 0,481^{\mathrm{aA}}$ & $1,625 \pm 1,134^{a c}$ & $1,500 \pm 0,510^{a 8}$ \\
\hline
\end{tabular}

*Different superscript letters indicate statistically difference at $5 \%$ significant level $(p<0.05)$. ( $a, b, c$ for rows and $A, B, C$ for columns)

Comparing the image quality of repair materials, no difference was observed among the repair materials $(p>0.05)$. Estimated marginal means are shown in Figure 1.

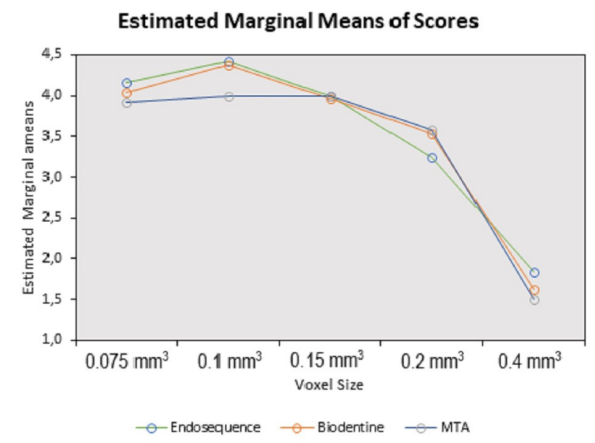

Figure 1. Estimated marginal means of scores according to voxel sizes.

\section{DISCUSSION}

Clinicians should be able to manage the case well when the perforation, which is an undesirable complication occurs. In addition to choosing the most appropriate material to be used, post-treatment radiographic control is also important. Therefore, the present study investigated which voxel setting would be necessary to get the best visual for imaging the material. Conventional periapical radiographs have limitations for evaluation of repair material quality. CBCT is used in many areas in endodontics and has been shown to have the greatest accuracy among imaging modalities for perforation detection (13). Although it has many clinical advantages, including 3D visualization, CBCT has some limitations. The most important limitation is the radiation exposure. The goal is to obtain the best image with a minimum radiation dose. Effective dose differs between CBCT scanners; depending on the size of the FOV, it may be similar to panoramic radiography, but significantly less than medical CT. Image acquisition with a larger voxel size followed by reconstruction at a smaller voxel size may yield similar quality with a reduced radiation dose (14).

Few studies evaluated voxel sizes for furcation perforations. Tomographic image quality is related directly to the acquisition protocol, and particularly the voxel size. Junqueira et al. (15) used voxels of the least thicknesses available (250 and 125 micron) because a lesser slice thickness generates greater spatial resolution, which can directly affect the diagnostic capability. Although they found no significant difference between examinations, the sensitivity and accuracy were less with larger voxels, supporting the results described by Silveira et al. (16), Melo et al. (17) and BritoJúnior et al. (18). Filling materials were evaluated on images acquired with 200 micron voxels. Properties of all filling materials were similar on images obtained with a 76 micron voxel size. Properties differed among voxel sizes only for Sealer 26 and Endofill, with more artifacts observed with 200 micron voxel resolution (18). Similarly, in our study, image quality was low and artifacts were observed with 200 and 400 micron. Liedke et al. (19) and Silveria et al. (16) assessed the performance for external root resorption, found that the 300 micron voxel size yielded the best outcomes, with less exposures. In our study, the best image quality for furcation defects was achieved with 100 micron voxels. In a previous study in which mandibular molar morphology was examined using $\mathrm{CBCT}$, the best voxel size was determined to be 100 micron (20). Other in vitro studies have demonstrated that $\mathrm{CBCT}$ is more reliable than conventional radiography for the diagnosis of endodontic pathologies, such as root fracture, root perforation, and resorption (21). Venskutonis et al. (22) stated in their study for the detection of root perforations the reduction of the voxel size increased the diagnostic value.

Another major disadvantage of CBCT is the appearance of restorative material artifacts, which can be caused by most materials used in endodontics. The restorative materials have specific densities can create artifacts such as blooming, scatter streaks, which can consequence for false-positive results. Thus, the accuracy of CBCT evaluation of root-filled teeth can be compromised by the presence of artifacts such as noise, scattering, missing values due to motion, and rings, as well as beam-hardening and streaking artifacts, which complicate image interpretation (12). Despite these disadvantages, CBCT is the chosen imaging modality for root canal perforations, furcation perforations, and other pathologies. In this study, the visibility of furcation perforations and were evaluated on $\mathrm{CBCT}$ images (Figure 2). 


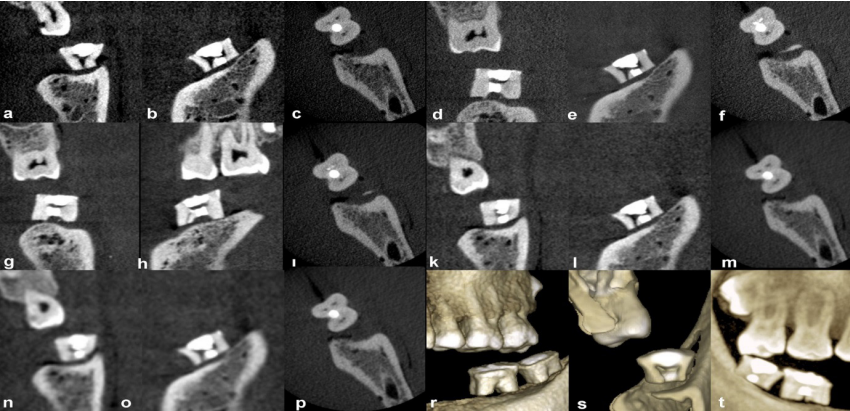

Figure 2. $a, b$ and $c$ : $C B C T$ images show cross section, sagittal, and axial of 75 micron furcation defects with sealing material. $d$, e and $f$ : CBCT images show cross section, sagittal, and axial of 100 micron furcation defects with sealing material. $g$, $h$ and $I: C B C T$ images show cross section, sagittal, and axial of 150 micron furcation defects with sealing material. $k$, I and m: CBCT images show cross section, sagittal, and axial of 200 micron furcation defects with sealing material. $n$, $o$ and $p$ : CBCT images show cross section, sagittal, and axial of 400 micron furcation defects with sealing material. $r$, $s$ and $t$ : CBCT images show 3D representation of furcal defects from buccal cross section and lingual slides.

A perforation - an iatrogenic, mechanical, or compulsive communication between the root canal system and external tooth surface - should be sealed with a biocompatible material. Jeevani et al. (23) reported that MTA is now considered to be the material of choice for perforation sealing because of its biocompatibility. However, MTA has shortcomings like trouble of handling and slow setting, which may subsidize to leakage, surface disintegration, loss of marginal adaptation, and disruption of the material. As stated before, during image evaluation of various materials, Brito-Júnior et al. (18) assessed streaking artifacts produced by Sealer-26, Endofill, and MTA FillApex. They found Sealer-26 and Endofill produced more artifacts than did MTA FillApex at a voxel size of 200 micron. Biodentine is a calcium silicate-based material that has a polycarboxylate-based hydrosoluble polymer and contains Calcium cilicate. The latter has high compressive and flexural strength. Helvacioglu et al. (12) reported that Biodentine created the fewest artifacts and showed the best performance among materials evaluated. EndoSequence was developed to overcome the difficulty of handling of MTA. No previous study has explored the $\mathrm{CBCT}$ visualization or artifact formation of EndoSequence. No significant difference in image clarity was found among materials in this study. Of five voxel sizes, 100 micron yielded the clearest images.

The limitation of this study was in clinical practice detection of furcal defects in CBCT machines may be less accurate owing metallic or other artifacts. Moreover, patient movement should also be taken into consideration which in this study did not taken into account because of the in vitro nature of the study. Thus, further studies should be done with CBCT optimization filters.

\section{CONCLUSION}

As a consequence of this study $\mathrm{CBCT}$ imaging can be used to examine endodontic repair materials. A voxel size of 150 micron was recognized as the cut-off point for exposure of furcal defects repairing materials.

\section{Conflict of interest}

The authors declare that they have no conflict of interest.

\section{REFERENCES}

[1] da Fonseca TS, Silva GF, Guerreiro-Tanomaru JM, Delfino MM, Sasso-Cerri E, Tanomaru-Filho M, Cerri PS. Biodentine and MTA immunoinflammatory response favoring bone formation in sealing of furcation perforations in rat molars. Clin Oral Investig. 2019;23:1237-1252.

[2] Kamburoğlu K, Yeta EN, Yılmaz F. Ex vivo comparison of diagnostic accuracy of cone-beam computed tomography and periapical radiography in the detection of furcal perforations. J Endod. 2015;41:696-702.

[3] Sinai IH. Endodontic perforations: Their prognosis and treatment. J Am Dent Assoc 1977; 95:90-95.

[4] Camilo do Carmo Monteiro J, Rodrigues Tonetto M, Coêlho Bandeca M, Henrique Borges A, Cláudio Martins Segalla J, Cristina Fagundes Jordão-Basso K, Fernando Sanchez-Puetate C, Carlos Kuga M. Repair of iatrogenic furcal perforation with Mineral Trioxide Aggregate: A seven-year follow-up. Iran Endod J. 2017;12:516-20.

[5] de Oliveira NG, de Souza Araújo PR, da Silveira MT, Sobral APV, Carvalho MV. Comparison of the biocompatibility of calcium silicate-based materials to Mineral Trioxide Aggregate: Systematic review. Eur J Dent. 2018;12:317-326.

[6] Jitaru S, Hodisan I, Timis L, Lucian A, Bud M. The use of bioceramics in endodontics - literature review. Clujul Med. 2016;89:470-473.

[7] Gandolfi MG, lezzi G, Piattelli A, Prati C, Scarano A.Osteoinductive potential and bone-bonding ability of ProRoot MTA, MTA Plus and Biodentine in rabbit intramedullary model: Microchemical characterization and histological analysis. Dent Mater. 2017;33:e221-e238.

[8] Tang JJ, Shen ZS, Qin W, Lin Z. A comparison of the sealing abilities between Biodentine and MTA as root-end filling materials and their effects on bone healing in dogs after periradicular surgery. J Appl Oral Sci. 2019;7:27-e20180693.

[9] Abu Zeid ST, Alamoudi RA, Abou Neel EA, Mokeem Saleh AA. Morphological and spectroscopic study of an apatite layer induced by fast-set versus regular-set endosequence root repair materials. Materials (Basel) 2019;8:12-22.

[10] Celikten B, Jacobs R, de Faria Vasconcelos K, Huang Y, Shaheen $\mathrm{E}$, Nicolielo LFP, Orhan K. Comparative evaluation of cone beam CT and micro-CT on blooming artifacts in human teeth filled with bioceramic sealers. Clin Oral Investig. 2019;23:3267-3273.

[11] Braun X, Ritter L, Jervøe-Storm PM, Frentzen M. Diagnostic accuracy of CBCT for periodontal lesions. Clin Oral Investig. 2014;18:1229-1236.

[12] Helvacioglu-Yigit D, Demirturk Kocasarac H, Bechara B, Noujeim M. Evaluation and reduction of artefacts generated by 4 different root-end filling materials by using multiple cone-beam computed tomography imaging settings. J Endod. 2016;42:307-314. 
[13] Shokri A, Eskandarloo A, Noruzi-Gangachin M, Khajeh S. Detection of root perforations using conventional and digital intraoral radiography, multidetector computed tomography and cone beam computed tomography. Restor Dent Endod. 2015;40:58-67.

[14] Venskutonis T, Plotino G, Juodzbalys G, Mickevičienė L. The importance of cone-beam computed tomography in the management of endodontic problems: A review of the literature. J Endod. 2014;40:1895-1901.

[15] Junqueira RB, Verner FS, Campos CN, Devito KL, do Carmo $A M$. Detection of vertical root fractures in the presence of intracanal metallic post: A comparison between periapical radiography and cone-beam computed tomography. J Endod. 2013;39:1620-1624.

[16] Silveira PF, Vizzoto MB, Liedke GS, da Silveira HL, Montagner F, Silveira HE. Detection of vertical root fractures by conventional radiographic examination and cone beam computed tomography: An in vitro analysis. Dent Traumatol. 2013;29:41-46.

[17] Melo SLS, Bortoluzzi EA, Abreu M Jr, Corrêa LR, Corrêa M. Diagnostic ability of a cone-beam computed tomography scan to assess longitudinal root fractures in prosthetically treated teeth. J Endod. 2010;36:1879-1882.
[18] Brito-Júnior M, Santos LA, Faria-e-Silva AL, Pereira RD, SousaNeto MD. Ex vivo evaluation of artefacts mimicking fracture lines on cone-beam computed tomography produced by different root canal sealers. Int Endod J. 2014;47:26-31

[19] Liedke GS, da Silveira HE, da Silveira HL, Dutra V, de Figueiredo JA. Influence of voxel size in the diagnostic ability of cone beam tomography to evaluate simulated external root resorption. J Endod. 2009;35:233-235.

[20] Asgary S, Nikneshan S, Akbarzadeh-Bagheban A, Emadi $N$. Evaluation of diagnostic accuracy and dimensional measurements by using CBCT inmandibular first molars. J Clin Exp Dent. 2016;1:8e1-8.

[21] Lo Giudice R, Nicita F, Puleio F, Alibrandi A, Cervino G, LizioAS, Pantaleo G. Accuracy of periapical radiography and $\mathrm{CBCT}$ in endodontic evaluation. Int J Dent. 2018; 2018:2514243.

[22] Venskutonis T, Juodzbalys G, Nackaerts O, Mickevicienè L. Influence of voxel size on the diagnostic ability of conebeam computed tomography to evaluate simulated root perforations. Oral Radiol. 2013;29:151-159.

[23] Jeevani E, Jayaprakash T, Bolla N, Vemuri S, Sunil CR, Kalluru RS. Evaluation of sealing ability of MM-MTA, Endosequence, and biodentine as furcation repair materials: UV spectrophotometric analysi. J Conserv Dent. 2014;17:340-343.

How to cite this article: Orhan Al, Tufenkci P, Oncu A, Sevgi S, Celikten B, Orhan K.CBCT Visualization of Furcation Perforation Repair Materials Using Different Voxel Sizes. Clin Exp Health Sci 2021; 11: 654-658. DOI: 10.33808/ clinexphealthsci.954463 